\title{
Microendoscopic Excision of Osteoid Osteoma in the Pedicle of the Third Lumbar Vertebra
}

\author{
Katsuhito Yoshioka ${ }^{1}$, Eizo Matsuda $^{2}$, Hideki Murakami ${ }^{1}$, Hiroyuki Tsuchiya ${ }^{1}$ \\ ${ }^{1}$ Department of Orthopaedic Surgery, Graduate School of Medical Science, Kanazawa University, Kanazawa, Japan \\ ${ }^{2}$ Department of Orthopaedic Surgery, Houju Memorial Hospital, Nomi, Japan
}

We present a rare case of a patient who underwent complete microendoscopic excision of an osteoid osteoma, which induced radiculopathy without nerve root compression. A 20-year-old man presented severe right groin pain that was temporarily relieved by nonsteroidal anti-inflammatory drugs. A computed tomography (CT) scan showed typical features of a nidus located in the inferior cortex of the right $\mathrm{L} 3$ pedicle. We performed surgery using a posterior microendoscopic approach. We drilled vertically along the line of the cortex of the caudal pedicle using a high-speed drill. After identifying the tumor, en bloc resection of the nidus was achieved. Immediately after surgery, pain in the right groin disappeared. A CT scan showed that most of the right L3 pedicle remained. This minimally invasive technique preserves spinal structures, including the facet and pedicle, and is a viable option for the treatment of spinal osteoid osteomas located close to vital structures.

Keywords: Osteoid osteoma; Endoscopic surgery; Minimally invasive surgical procedure; Radiculopathy

\section{Introduction}

Osteoid osteoma is a small benign bone tumor that is commonly localized in long bones. However, in $10 \%$ of cases, it occurs in the spine. The majority of spinal osteoid osteomas are found in the posterior elements of the vertebrae [1]. The most common presenting symptom is pain that worsens at night and is relieved by aspirin. The curative approach for osteoid osteoma is operative removal or deactivation of the nidus with coagulation [2]. However, if the treatment is not complete, pain will persist and additional operative intervention will be necessary [3]. In recent years, minimally invasive techniques have been introduced in the management of spinal osteoid osteoma, such as percutaneous radiofrequency ablation (RFA) and anterior endoscopic excision of a tumor located in the C2 vertebral body [4-9]. We report the complete excision of an osteoid osteoma that induced radiculopathy without nerve root compression using a posterior microendoscopic approach.

\section{Technical Note}

A 20-year-old man presented with a 3-week history of severe right groin pain that was temporarily relieved by nonsteroidal anti-inflammatory drugs. Although he underwent examination at the internal medicine and urology departments, the cause of his pain could not be determined. He consulted the orthopedics department because of additional low back pain. The physical examination revealed a severe limitation in his range of motion, which was exacerbated by pain, but he emphasized that the pain

\footnotetext{
Received Jan 7, 2015; Revised Jan 29, 2015; Accepted Feb 12, 2015

Corresponding author: Katsuhito Yoshioka

Department of Orthopaedic Surgery, Graduate School of Medical Science, Kanazawa University,

13-1 Takara-machi, Kanazawa, 920-8641, Japan

Tel: +81-76-265-2374, Fax: +81-76-234-4261, E-mail: ortho0825yoshy@yahoo.co.jp
} 
was more severe in the right groin than in the lower back. However, the results of his neurological examination were normal. No abnormalities could be found aside from a Cobb angle of 8 degrees, indicating scoliosis, on conventional radiography. A computed tomography (CT) scan demonstrated typical features of a nidus located in the inferior cortex of the right L3 pedicle (Fig. 1). The nidus size was $5 \mathrm{~mm}$. The tumor did not exceed the pedicle. Magnetic resonance imaging (MRI) showed inflammatory changes in the entire right pedicle and the right $\mathrm{L} 3 / 4$ intervertebral foramen (Fig. 2). However, the tumor did not compress the nerve root or the spinal cord. Clinicoradiological data indicated osteoid osteoma.
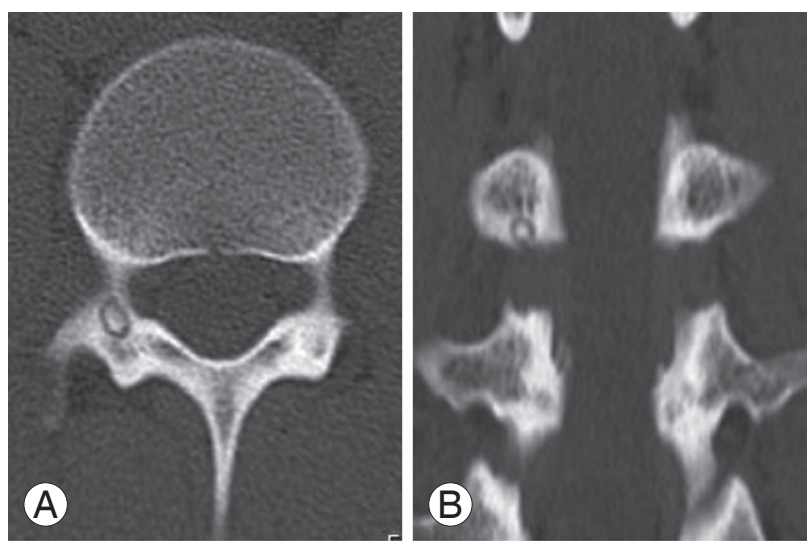

Fig. 1. Computed tomography scan revealed typical features of a nidus located in the inferior cortex of the right $\mathrm{L} 3$ pedicle. (A) Axial view, (B) coronal view.

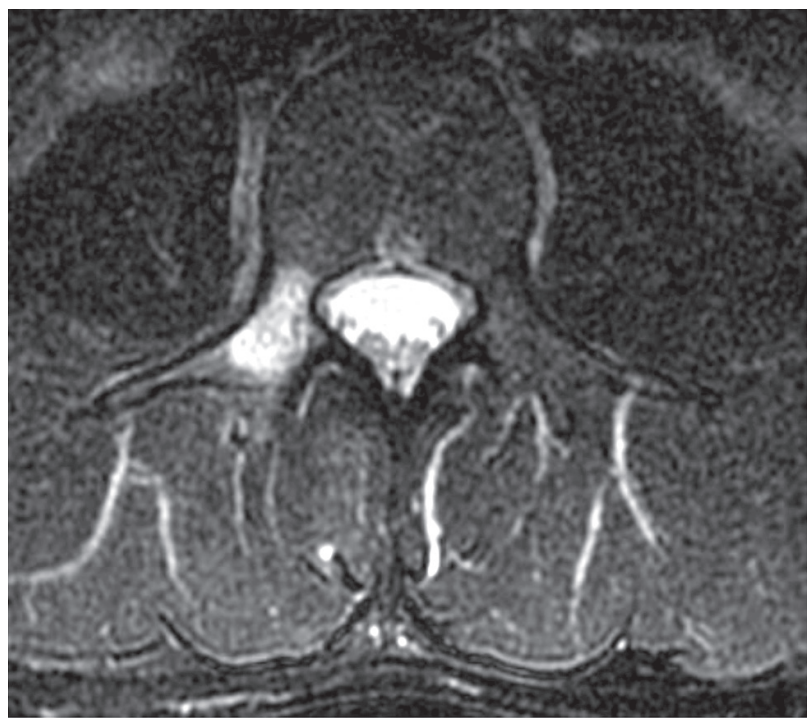

Fig. 2. Axial magnetic resonance imaging demonstrates inflammatory changes in the entire right pedicle.
We performed surgery using a posterior microendoscopic approach. Fluoroscopy was performed preoperatively to locate the exact point for introduction of the tubular retractors (Medtronic Sofamor Danek, Memphis, TN, USA). The $18-\mathrm{mm}$ skin incision was made $20 \mathrm{~mm}$ lateral to the spinous process, and the muscle was sequentially dilated after fasciotomy. The accessory process was used as a landmark, and a 16-mm-diameter tubular retractor was placed on the right pedicle. The location of the $\mathrm{L} 2 / 3$ facet or the transverse process did not need to be exposed. The inferior edge of the pedicle was confirmed, and we drilled vertically along the line of the cortex of the caudal pedicle using a 3-mm high-speed drill. The nidus in the cortex of the pedicle was identified, and en bloc resection was achieved (Fig. 3A). The right $\mathrm{L} 3$ nerve root
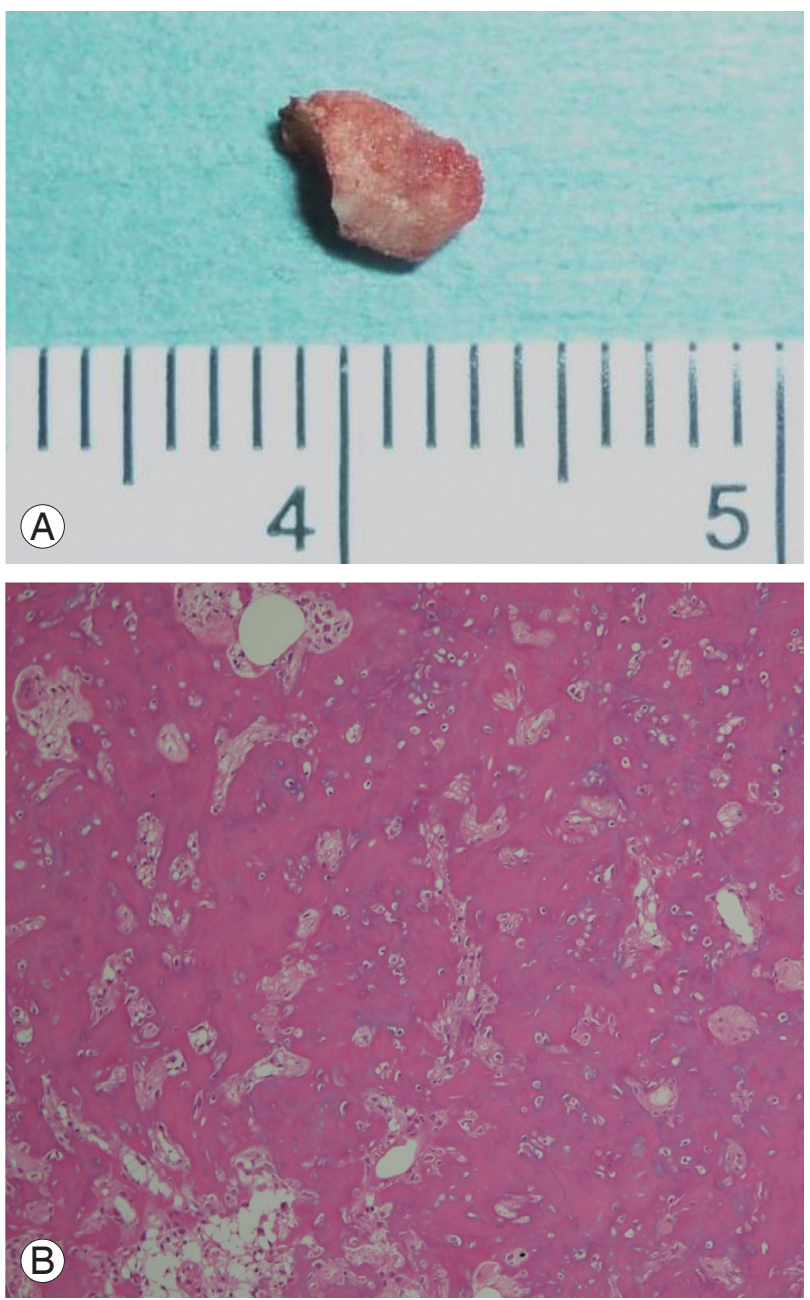

Fig. 3. We performed en bloc resection of the nidus (A). Histologic analysis of the specimens revealed rich fibrovascular stroma, prominent osteoblasts, and osteoid tissue, confirming the diagnosis of osteoid osteoma (B). H\&E, $\times 100$. 
was almost in contact with the osteoid osteoma, but no compression was observed.

Immediately after surgery, pain in the right groin disappeared disappeared. No surgery-related complications were observed. Histologic analysis of the specimens revealed rich fibrovascular stroma, prominent osteoblasts, and osteoid tissue, confirming the diagnosis of osteoid osteoma (Fig. 3B). A postoperative CT scan showed that only the nidus was excised completely and that most of the pedicle remained intact (Fig. 4). At the 2-year followup examination, the patient was asymptomatic with no recurrence.

\section{Discussion}

It is difficult to detect osteoid osteoma in the lumbar spine on radiography due to small tumor size and the overlap of intestinal gas on the lumbar spine. Davies et al. [10] reported that osteoid osteomas have a highly variable appearance on MRI, and reliance on MRI, including gadolinium-enhanced images, may lead to misdiagnosis. MRI showed reactive changes in the bone marrow and soft tissues, but could not detect the nidus in our case. Alternatively, the CT scan is generally regarded as the preferred cross-sectional technique for confirming osteoid osteoma. Our patient was diagnosed based on characteristic clinical features and the CT image.

Although osteoid osteoma often develops close to the spinal cord and nerve roots, due to the small size of the lesion, neurologic impairment rarely occurs $[1,11,12]$. Only one report detailed radiculopathy without nerve root compression from osteoid osteoma [12]. Neurological symptoms may be induced by tumorous inflammation even without nerve root compression. In the present case, the right L3 root was next to the tumor, and the patient's lower back and groin pain disappeared after surgical resection. It is possible that the patient's groin pain was referred pain; however, MRI indicated inflammatory changes in the right L3/4 intervertebral foramen. These findings suggest the possibility that $\mathrm{L} 3$ radiculopathy from tumorous inflammation caused his groin pain. L3 radiculopathy usually results in anterior thigh pain instead of the groin pain experienced by this patient. However, Hirabayashi et al. [13] reported that groin pain was observed in $18 \%$ of L3 radiculopathy cases. This report also supported groin pain as a result of the patient's L3 radiculopathy.

Osteoid osteoma of the spine requires entire removal of the nidus. Surgical open exposure can disrupt the muscle and bony elements that are important to stability, resulting in a limitation in the mobility of the spinal column secondary to fusion using instrumentation. En bloc resection of the spinal lesion could lead to instability in young patients, especially in the pedicle as in this case. Conversely, RFA is the most commonly used minimally invasive method for osteoid osteoma $[2,4,5,9]$. Recent studies reported that RFA is a possible treatment for osteoid osteomas located close to $(<10 \mathrm{~mm})$ vital structures, such as arteries, the spinal cord, and nerve roots, without complications [6]. However, surgical treatment is recommended for lesions that are located adjacent $(<2 \mathrm{~mm})$ to the nerve root [6]. We considered RFA to be inappropriate for the present case. Another problem is that histologic evidence
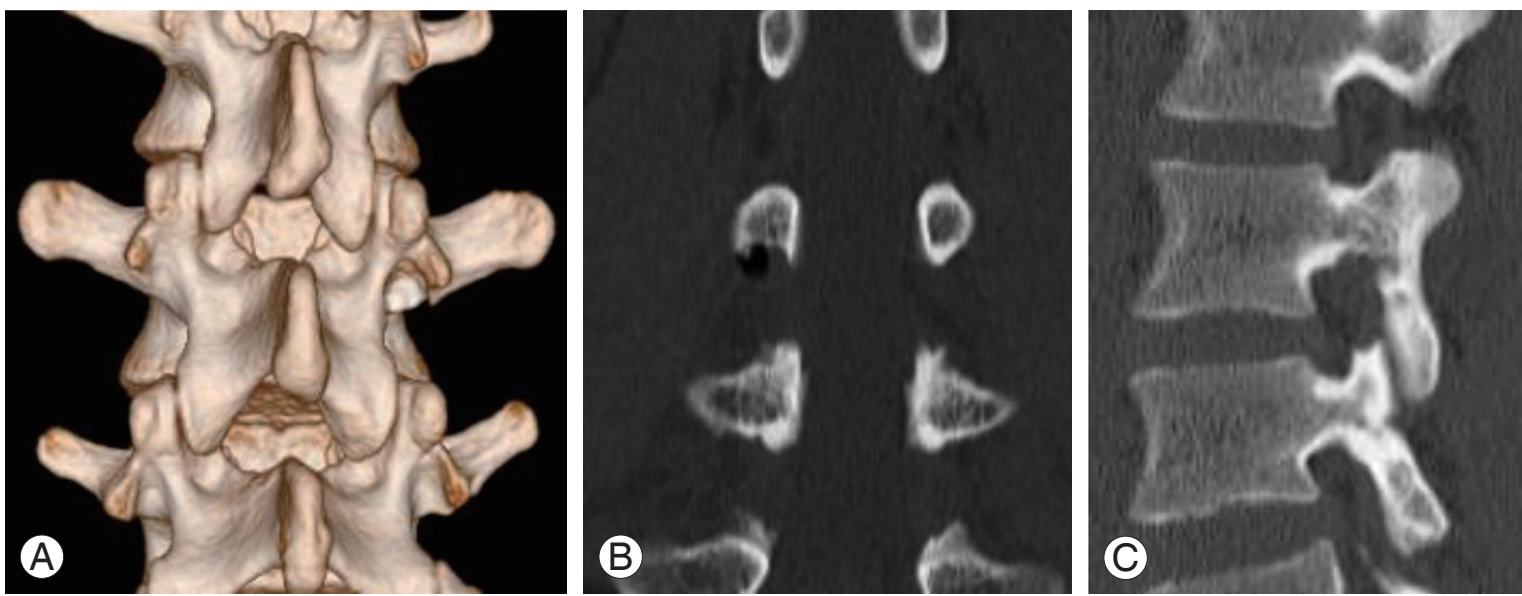

Fig. 4. A postoperative computed tomography scan showed that only the nidus was excised completely and that most of the pedicle remained intact. (A) 3-Dimensional-computed tomography, (B) coronal view, (C) sagittal view. 
of the osteoid osteoma is impossible to recover when using RFA. Although our spinal microendoscopic surgery required knowledge of deep anatomical structures, meticulous preoperative planning, and a learning curve, this procedure had the following advantages: small skin incision, shorter hospitalization, shorter rehabilitation, preserved facet and pedicle, no need for fusion, and clear confirmation of the small tumor. Additionally, unique visualization through an oblique lens $\left(25^{\circ}\right)$ helped identify the orientation of the nerve root, pedicle, and tumor.

We described a rare case of osteoid spinal osteoid osteoma, which induced radiculopathy without nerve root compression. The nidus was completely resected without complication using a microendoscope. This minimally invasive technique preserves spinal structures including the facet and pedicle.

\section{Conflict of Interest}

No potential conflict of interest relevant to this article was reported.

\section{References}

1. Gasbarrini A, Cappuccio M, Bandiera S, Amendola L, van Urk P, Boriani S. Osteoid osteoma of the mobile spine: surgical outcomes in 81 patients. Spine (Phila Pa 1976) 2011;36:2089-93.

2. Rosenthal DI, Hornicek FJ, Wolfe MW, Jennings LC, Gebhardt MC, Mankin HJ. Percutaneous radiofrequency coagulation of osteoid osteoma compared with operative treatment. J Bone Joint Surg Am 1998; 80:815-21.

3. Lee DH, Malawer MM. Staging and treatment of primary and persistent (recurrent) osteoid osteoma. Evaluation of intraoperative nuclear scanning, tetracycline fluorescence, and tomography. Clin Orthop Relat Res 1992;(281):229-38.
4. Rehnitz C, Sprengel SD, Lehner B, et al. CT-guided radiofrequency ablation of osteoid osteoma and osteoblastoma: clinical success and long-term follow up in 77 patients. Eur J Radiol 2012;81:3426-34.

5. Cioni R, Armillotta N, Bargellini I, et al. CT-guided radiofrequency ablation of osteoid osteoma: longterm results. Eur Radiol 2004;14:1203-8.

6. Vanderschueren GM, Obermann WR, Dijkstra SP, Taminiau AH, Bloem JL, van Erkel AR. Radiofrequency ablation of spinal osteoid osteoma: clinical outcome. Spine (Phila Pa 1976) 2009;34:901-4.

7. Rybak LD, Gangi A, Buy X, La Rocca Vieira R, Wittig J. Thermal ablation of spinal osteoid osteomas close to neural elements: technical considerations. AJR Am J Roentgenol 2010;195:W293-8.

8. Amendola L, Cappuccio M, Boriani L, Gasbarrini A. Endoscopic excision of C2 osteoid osteoma: a technical case report. Eur Spine J 2013;22 Suppl 3:S357-62.

9. Laus M, Albisinni U, Alfonso C, Zappoli FA. Osteoid osteoma of the cervical spine: surgical treatment or percutaneous radiofrequency coagulation? Eur Spine J 2007;16:2078-82.

10. Davies M, Cassar-Pullicino VN, Davies AM, McCall IW, Tyrrell PN. The diagnostic accuracy of MR imaging in osteoid osteoma. Skeletal Radiol 2002;31:55969.

11. Kawaguchi Y, Hasegawa T, Oka S, Sato C, Arima N, Norimatsu H. Mechanism of intramedullary high intensity area on $\mathrm{T} 2$-weighted magnetic resonance imaging in osteoid osteoma: a possible role of COX-2 expression. Pathol Int 2001;51:933-7.

12. Zenmyo M, Yamamoto T, Ishidou Y, Komiya S, Ijiri $\mathrm{K}$. Osteoid osteoma near the intervertebral foramen may induce radiculopathy through tumorous inflammation. Diagn Pathol 2011;6:10.

13. Hirabayashi $\mathrm{H}$, Takahashi J, Hashidate H, et al. Characteristics of L3 nerve root radiculopathy. Surg Neurol 2009;72:36-40. 